\title{
ESTUDIO COMPARATIVO DEL SISTEMA PENSIONAL CHILE - COLOMBIA. MODELOS PENSIONALES, TIPOS DE PENSIÓN Y DESAFÍOS
}

\author{
Jeniffer Carolina Acuña Páez \\ Facultad de Negocios, Gestión y Sostenibilidad \\ Especialización en Gestión Empresarial \\ Jeacunap1@poligran.edu.co \\ Sandra Milena Suarez Macías \\ Facultad de Negocios, Gestión y Sostenibilidad \\ Especialización en Gestión Empresarial \\ Sasuarez10@poligran.edu.co
}

\section{Resumen}

Los sistemas de seguridad social en un país están generados para buscar una cobertura social al llegar a la edad de pensión, sin embargo, en algunos casos no se ha logrado cumplir con las expectativas de cobertura y el acceso a las prestaciones económicas manteniendo una sostenibilidad en el tiempo.

Por medio del presente artículo, se busca realizar una comparación entre los modelos pensionales de Colombia y Chile presentando las diferencias más relevantes entre los fondos privados y el sistema público, analizando los puntos a favor de cada sistema y los desafíos que enfrenta cada país. Parte de los argumentos que se presentan corresponden a las inquietudes que se generan al hacer un estudio sobre la viabilidad pensional en la población adulta de los países objeto de estudio.

Palabras claves: Pensión, Modelos pensionales; Sostenibilidad; Población; Sistema; Viabilidad. 


\section{Introducción}

Por medio del presente artículo se abordará un análisis entre los sistemas pensionales de Chile y Colombia con el fin de conocer los beneficios y las obligaciones normativas y constitucionales de cada país. Como base se toma la explicación de los regímenes tanto de Chile como de Colombia y se concluye con una breve explicación de las reformas pensionales propuestas.

Para contextualizar y brindar una mayor perspectiva sobre el sistema pensional, es necesario hacer un recorrido histórico a fin de entender como inicio la seguridad social. Hace alrededor de 120 años nace en el ámbito mundial la inquietud de generar un sistema para entregar jubilaciones a los adultos mayores y que fuera sostenible en el largo plazo, esto con el propósito de brindar bienestar a los grupos de trabajadores en su vida laboral activa. (Croquevielle, 2016).

Alemania fue el primer país en dar inicio a la creación de un sistema pensional (Croquevielle, 2016) ya que tras, la primera guerra mundial la protección social se volvió un tema de impacto generando la creación de diferentes organizaciones como lo fue la OIT. En el año de 1944 se generó la primera declaración de la OIT en cuanto a temas de seguridad social, en la cual la idea principal era el estudio social de los problemas que genera la administración de los sistemas de pensiones. Al siguiente año, se adoptó la Declaración de los Derechos Humanos, art. 22 donde se reconoce "Toda persona, como miembro de la sociedad, tiene derecho a la seguridad social" (Asamblea General de la ONU, 1948).

Desde este punto de vista las economías mundiales brindaron una definición desde la parte social y humana estructurando la seguridad social como un derecho y creando un sistema basado en cotizaciones con el cual se otorgaba a las personas garantías en salud, pensión y desempleo por casusas como pérdida de capacidad laboral, muerte, vejez o invalidez (OIT, 1996-2018). A nivel mundial, los sistemas pensionales se definen de acuerdo con la legislación de cada país, sin dejar de lado que todos tienen elementos en común. De esta forma, los sistemas pensionales se dividen o clasifican en tres categorías:

\begin{tabular}{|c|l|}
\hline \multicolumn{2}{|c|}{ Sistemas Pensionales } \\
\hline Reparto & $\begin{array}{l}\text { - Es un fondo común de ahorro para pensión administrado por el Estado. } \\
\text { - Se sostiene por aportes de los trabajadores activos que subsidian a los } \\
\text { pensionados. } \\
\text { - Dinamarca se rige por este sistema. }\end{array}$ \\
\hline $\begin{array}{c}\text { Capitalización } \\
\text { Individual }\end{array}$ & $\begin{array}{l}\text { - Los aportes son mensuales por cada trabajador durante su vida productiva. } \\
\text { - Cuando el trabajador llega a su edad de jubilación el beneficio depende de } \\
\text { lo que haya ahorrado durante el tiempo activo más la rentabilidad generada. } \\
\text { - Chile, Perú y Australia se rigen por este sistema. }\end{array}$ \\
\hline Mixto & $\begin{array}{l}\text { En este sistema se combinan los aportes realizados al Estado con los aportes } \\
\text { realizados en las instituciones privadas conocidas como AFP. }\end{array}$ \\
\hline
\end{tabular}

Nota: información recopilada de "Mis finanzas para invertir de Davivienda" (Davivienda, 2018)

Tabla 1. Sistemas pensionales. 
En Colombia el concepto de pensión se vuelve legal y obligatorio a partir de 1944 año en el cual este derecho se enmarca en un conjunto de acciones y normas en las cuales se establecerían las relaciones entre la nación y los empleadores. De acuerdo a lo anterior se crea un sistema específico de pensiones iniciando con el primer régimen pensional Colombiano denominado Cajanal " el cual se inició en 1945 con la Ley 6 y fue modificado por la Ley 90 de 1946 con la cual se creó el ISS(Instituto de Seguros Sociales)" Este régimen estaba enfocado en brindar garantías a los empleados públicos pero presento deficiencias debido a la estrategia de planeación la cual consistía en un aumento gradual por cada periodo de 5 años del ingreso base de cotización (Fedesarrollo, 2010). Debido a la falta de estrategia el aumento gradual propuesto no se cumplió por lo tanto el gobierno acepto que el ingreso base de cotización se mantuviera estable durante 16 años efectuando un aumento de dos puntos en el año de 1985 y volviendo a dejar constante el ingreso por un periodo de diez años. Otro de los factores en contra de este sistema fue el cambio demográfico ya que la población colombiana empezó a envejecer disminuyendo la cantidad de cotizantes activos y aumentando la cantidad de pensionados. Se espera que para el año 2050 la población mayor aumente tres veces con respecto a la de 1975 "información basada en la reunión anual de seguimiento presentada por la AFP Porvenir" (Porvenir, s.f.).

Al ser un sistema piramidal afecto la estructura del modelo pensional llevándolo a una restructuración buscando un sistema que ampliara la cobertura a toda la población y que controlara los vacíos generados ajustando las exigencias en las cotizaciones y los requisitos para la obtención de la pensión.

En cuanto al modelo pensional de Chile su modelo plantea un sistema solidario que ofrece beneficios a su población más vulnerable. De acuerdo a su normatividad la población chilena podrá tener su pensión si son mayores de 65 años y componen un grupo familiar perteneciente al $60 \%$ de personas más pobres con lo que se ofrece un mínimo vital para la supervivencia.

Como se evidencia en lo anteriormente descrito se hace énfasis en el modelo pensional colombiano ya que este es un sistema basado en la experiencia chilena. Donde es básico buscar alternativas de mejora ya que Colombia cuenta con varias falencias y necesita una intervención urgente.

En este enfoque para la recolección de la información se utilizan las fuentes de análisis de la normatividad legal para realizar una comparación de cada sistema, así mismo se consultaron páginas web, Artículos sobre pensión desarrollados por las instituciones expuestas en las referencias bibliográficas, presentaciones de los directivos de la AFP Porvenir, libros y documentos que abordan el tema de estudio.

La metodología con la que se realizó este artículo (Figura 1. Acuña \& Suarez, 2018) se generó a partir de una investigación descriptiva con base en rastreo bibliográfico derivado de documentos de carácter científico abordando el tema de lo general a lo particular, iniciando con antecedentes 
históricos del sistema de seguridad social en el mundo y explicando las características propias de los sistemas pensionales de Chile y Colombia.

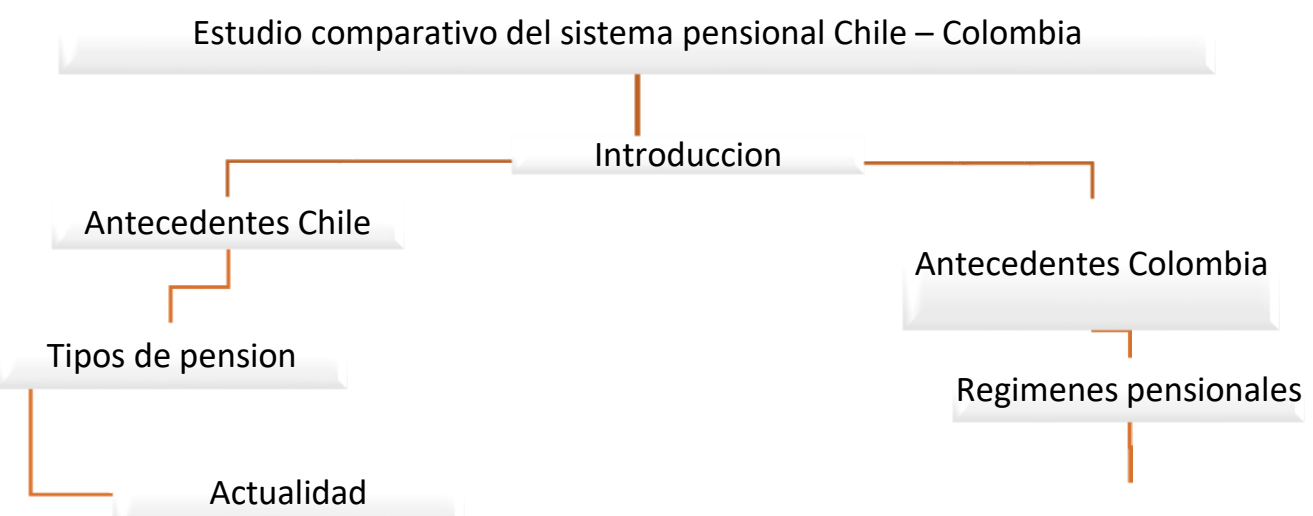

Tipos de pension

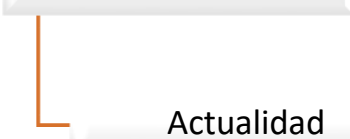

Figura 1. Diagrama de metodología. Elaboración propia (Acuña \& Suarez, 2018)

\section{Referente teórico}

\section{¿Que son las AFP?}

Las Administradoras de Fondos de Pensiones (AFP) nacen en el año 1980 y son sociedades anónimas que tienen objetivo administrar un fondo de pensiones y otorgar a sus afiliados las prestaciones que establece la ley. Se financian a través del cobro de comisiones a sus afiliados y podrán aumentar los ahorros de estos mediante inversiones (Biblioteca del Congreso Nacional de Chile, 2013)

Antes todas las cuentas individuales eran invertidas de la misma forma. No obstante, con el sistema de multifondos, las AFP separan los dineros en los fondos de inversión, con distinto riesgo capitalizador. El afiliado debe voluntariamente elegir uno de estos fondos según su interés de ganancia de rentabilidad. La idea es que el afiliado elija el tipo de inversión y nivel de riesgo en que se usarán sus fondos de acuerdo con cada país, a continuación, se nombraran cada uno de los de los sistemas de multifondos que se manejan: 


\begin{tabular}{|l|c|}
\hline \multicolumn{1}{|c|}{ Chile } & Colombia \\
\hline Fondo A: Más Riesgoso & Fondo Conservador \\
\hline Fondo B: Riesgoso & Fondo Moderado \\
\hline Fondo C: Intermedio & Fondo de Mayor Riesgo \\
\hline Fondo D: Conservador & Fondo Especial de Retiro Programado \\
\hline Fondo E: Más Conservador & - \\
\hline
\end{tabular}

Nota: Elaboración propia (Acuña \& Suarez, 2018)

Tabla 2. Portafolio de Multifondos Chile Colombia.

En este artículo se abordará el tema comparativo sobre los sistemas pensionales de los dos países y se dividirá entre la creación de las AFP, la sostenibilidad y los retos a futuro que se presentan al ser, en el caso de Chile pionero en el manejo de seguridad social en América Latina y Colombia como importador del modelo chileno. Adicionalmente, se considera la perspectiva social al tratarse de un tema primordial donde se logra acceder a los beneficios necesarios al llegar a la edad de jubilación.

En las AFP se encuentran 3 tipos de pensiones: Vejez, Invalidez y Sobrevivencia. (Rankia, 2016; Rankia, 2018).

AFP En cada uno de los países:

\begin{tabular}{|c|c|}
\hline ENTIDADES PENSIONALES CHILE & $\begin{array}{l}\text { ENTIDADES PENSIONALES COLOMBIA REGIMEN } \\
\text { DE AHORRO INDIVIDUAL (PRIVADO) }\end{array}$ \\
\hline AFP Capital & Porvenir \\
\hline AFP Cuprum Cuprum $A F$ & Protección \\
\hline AFP Habitat HABITAAP & Old Mutual gral oldmutual \\
\hline AFP Modelo ofp modelo & Colfondos \\
\hline AFP Planvital & $\begin{array}{l}\text { ENTIDAD PENSIONAL COLOMBIA REGIMEN DE } \\
\text { PRIMA MEDIA (PUBLICO) }\end{array}$ \\
\hline AFP Provida AFP Provida & Polpensiones \\
\hline
\end{tabular}

Nota: Elaboración propia (Acuña \& Suarez, 2018)

Tabla 3. Administradoras de Fondos de Pensiones Chile-Colombia 
Para finalizar este artículo nace como un medio de consulta en el cual se agrupa la normatividad, tipos de pensión, requisitos y diferencias tomando como referencia el sistema pensional chileno el cual cuenta con una destacada sostenibilidad en América latina.

\section{El sistema Pensional en Chile}

\section{Inicios del sistema pensional en Chile}

En América Latina, Chile es uno de los países pioneros en seguridad social, ya que se adentró en el sistema pensional durante las primeras décadas del siglo $\mathrm{XX}$.

En 1924 se genera una política pública de previsión social la cual se dirigía por medio de las Cajas de Previsiones las cuales manejaban sus condiciones de afiliación y cobertura, con este sistema se asignaba un monto del valor de la pensión; sin embargo, estas cajas se volvieron inviables y en el año de 1980 durante la dictadura chilena se fundó el "Modelo de capitalización individual" y es donde nacen la AFP (Asociación AFP Chile). Por medio del financiamiento personal cada trabajador se encarga de formar el capital para su jubilación. Como lo muestra la siguiente gráfica:

En 1981 se efectúa una reforma la cual-_generó una gran atención al ser un sistema exportado a diferentes países (entre ellos Colombia).

El sistema pensional de Chile está dividido en dos formas: Uno público no contributivo y uno contributivo obligatorio basado en el ahorro y la capitalización. Adicionalmente, las personas pueden realizar aportes voluntarios.

La prioridad del modelo pensional es buscar el bienestar de los trabajadores y tener el menor impacto fiscal y económico en el mercado de capitales del país.

Con las Administradoras de Fondos de Pensiones se abrió un campo de inversiones, el cual fue aprovechado con la venta y privatización de empresas estatales.

La Ley No 20255 estableció la reforma previsional, implementando 3 pilares básicos que conforman un sistema de pensiones (Ley No 20255 (Diario Oficial de la República de Chile, Santiago, Chile, 2008). la cual fue promulgada el 11 de marzo de 2008 y entró en vigencia a partir del 1 de Julio de 2008 (Ministerio de del Trabajo y Previsión Social de Chile (31 de agosto de 2011), donde se implantaron 3 pilares básicos que conforman un sistema de pensiones. 

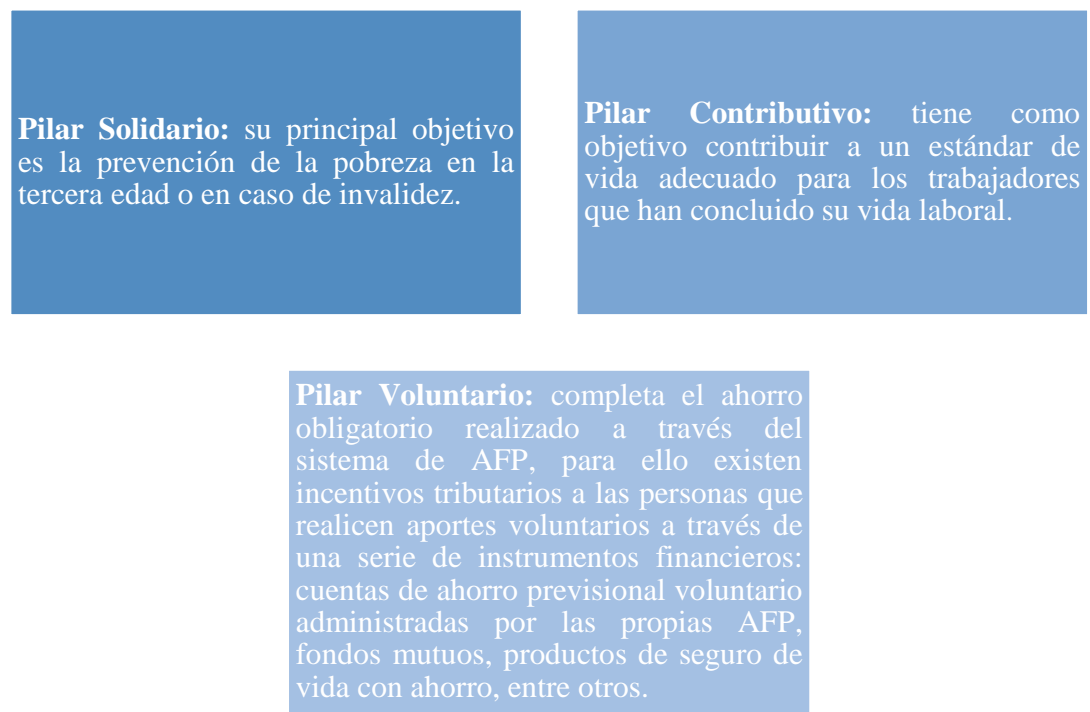

Figura 2. Pilares del Sistema Pensional en Chile. Diagrama elaborado con base a la información presentada en la VII Jornada de sistema de pensiones de Chile. (Superintendencia de Pensiones, Santiago, Chile: Pilares básicos del Sistema de Pensiones, 2011. https://www.spensiones.cl/portal/institucional/594/w3-article-7817.html.

\section{Tipos de pensiones en Chile}

"Pensión de Vejez, afiliados que cumplan con la edad legal, 65 años los hombres y 60 años las mujeres.

El monto de la pensión será financiado con los recursos que cada trabajador haya alcanzado en su cuenta previsional a lo largo de su vida laboral. Si se encuentra dentro del $60 \%$ más pobre de la población puede complementar su pensión con el Pilar Solidario.

Pensión de vejez anticipada, si el afiliado logra financiar una pensión igual o superior al $70 \%$ del promedio de los ingresos de los últimos 10 años de trabajo o que el monto de la pensión sea igual o superior al $80 \%$ de la pensión máxima con aporte solidario (PMAS) vigente a la fecha de la solicitud.

Pensión de vejez por trabajos pesados se obtiene una pensión de vejez con una edad inferior a la exigida por labores específicas.

Pensión de Invalidez es una pensión anticipada a la cual pueden acceder aquellos afiliados al Sistema, que por medio de una comisión médica de la Superintendencia de Pensiones lo declare como discapacitado por lo menos con un $50 \%$ de sus capacidades físicas o mentales.

Pensión de Sobrevivencia pensión anticipada que se entrega a los componentes del grupo familiar que tengan derecho del afiliado fallecido y que cumplan con los requisitos exigidos por la ley. 
Estos tipos de pensiones se pueden solicitar bajo alguna de las siguientes modalidades:

- Retiro Programado

- Renta Vitalicia Inmediata

- Renta Temporal con Renta Vitalicia Diferida

- Renta Vitalicia Inmediata con Retiro Programado

Además, el sistema otorga los siguientes beneficios:

- Beneficios Garantizados por el Estado

- Cuota Mortuoria

- Excedente de Libre Disposición

- Herencia" (Rankia, s.f)

\section{El sistema pensional en Colombia}

\section{Inicios del sistema pensional en Colombia}

Colombia es un país intermedio en seguridad social. El sistema pensional colombiano inicio en 1945 por la Caja de Previsión Nacional y el Instituto Colombiano de Seguros Sociales (ISS) (Universidad del Valle, 2012), por inconsistencia en los aportes de los afiliados y la existencia de regímenes especiales empezaron a sobrepasar la capacidad de financiamiento del sistema. Por todas esas falencias el Estado llevó a cabo una reforma en la década de los noventa, la cual es regida y controlada por la Ley 100 de 1993, su objetivo es garantizar que la población se va a encontrar amparada contra las dificultades que se puedan derivar de la vejez, invalidez o muerte, reconociéndose una pensión y prestaciones determinadas por Ley con algunas excepciones.

Franco, Oquendo y Berrio (Franco, Oquendo, \& Berrio, 2015) en su artículo "Posibles perspectivas del sistema de pensiones en Colombia" hacen un compilado de la normatividad que reglamento el sistema antes y después de la ley 100 de 1993 como lo podemos ver en las Figura 3. Normatividad pensional en Colombia antes de la Ley 100 de 1993. e iError! No se encuentra el origen de la referencia. en las cuales podemos ver que la reglamentación desde sus inicios fue altamente discriminatoria. 


\begin{tabular}{|c|c|c|c|}
\hline $\begin{array}{l}\text { Normatividad } \\
\text { Relacionada }\end{array}$ & Componente Pensional que reglamenta & Normatividad Relacionada & Componente Pensional que Reglamenta \\
\hline Ley 29 de 1905 & $\begin{array}{l}\text { Régimen pensional para magistrados principales de la Corte Suprema de } \\
\text { Justicia, mayores de } 60 \text { años, con la mitad del sueldo del último empleo } \\
\text { ejercido. }\end{array}$ & Ley 100 de 1993 & $\begin{array}{l}\text { Crea el sistema de seguridad social integral y el Sistema General de } \\
\text { Pensiones en Colombia. }\end{array}$ \\
\hline Ley 29 de 1912 & $\begin{array}{l}\text { Pensiones a favor de las viudas de los Presidentes de la República, o de } \\
\text { sus hijas solteras. }\end{array}$ & Ley 549 de 1999 & $\begin{array}{l}\text { Crea el FONPET, como fondo de pensiones de las entidades } \\
\text { teritoriales en Colombia. }\end{array}$ \\
\hline Ley 57 de 1915 & Reparaciones por accidentes de trabajo. & & Modfica el Sistema General de Pensiones y se adoptan disposiciones \\
\hline Ley 80 de 1916 & Establece limites a los beneficios de la Ley anterior. & Ley 797 de 2003 & sobre Regimenes exceptuados y especiales. \\
\hline Ley 40 de 1922 & $\begin{array}{l}\text { Pensiones para militares contagiados de lepra y a los médicos y empleados } \\
\text { que los atiendan }\end{array}$ & Ley 860 de 2003 & Modifica el Sistema General de Pensiones. \\
\hline Ley 86 de 1923 & $\begin{array}{l}\text { Dispone que todo empleado público nacional que adquiera enfermedad } \\
\text { laboral, tiene derecho a la mitad de su sueldo mensual durante } 6 \text { meses, }\end{array}$ & Acto Legislativo No.1 de 2005 & $\begin{array}{l}\text { Adiciona el Articulo } 48 \text { de la C.P. sobre la seguridad social como un } \\
\text { servicio público obligatorio. }\end{array}$ \\
\hline Ley 102 de 1927 & $\begin{array}{l}\text { Pensiones a favor de las viudas de los magistrados de la corte Suprema de } \\
\text { Justicia con más de } 20 \text { años de servicio. }\end{array}$ & & Crea Colpensiones como Empresa Industrial y Comercial del Estado, \\
\hline Ley 6 de 1945 & $\begin{array}{l}\text { Disposiciones sobre convenciones de trabajo, asociaciones profesionales, } \\
\text { confictos colectivos. Crea la Caja Nacional de Previsión social.(Cajanal) }\end{array}$ & Ley 1151 de 2007 & $\begin{array}{l}\text { quien administrará el Régimen de Prima Media con prestación } \\
\text { definida e institucionaliza el Sistema de Segunidad Social Integral. }\end{array}$ \\
\hline $\begin{array}{l}\text { Ley } 90 \text { de } 1946 \\
\text { Ley4 de } 1966\end{array}$ & $\begin{array}{l}\text { Creae Il Instituto colombiano de seguros Sociales. } \\
\text { Nuevos recursos para Cajanal, reajusta pensiones de jubilación e invalidez. }\end{array}$ & Ley 1371 de 2009 & $\begin{array}{l}\text { Establece la concurrencia para el pago del pensional de las } \\
\text { universidades Estatales del nivel nacional y territorial. }\end{array}$ \\
\hline
\end{tabular}

Figura 3. Normatividad pensional en Colombia antes de la Ley 100 de 1993. (Franco, Oquendo, \& Berrio, 2015)

Es por este motivo que el sistema general de pensiones está concebido de tal manera que en él subsisten dos regímenes que casi pudiera decirse son antagónicos, contradictorios y excluyentes. Por esta razón se afirma que el modelo pensional colombiano es uno de los típicos sistemas paralelos, en contraposición a los sistemas substitutivos y mixtos (RANGEL, 2017).

Estos dos regímenes solidarios excluyentes son: Régimen Solidario de Prima media y Régimen de Ahorro Individual con Solidaridad y se componen de tres pilares fundamentales.
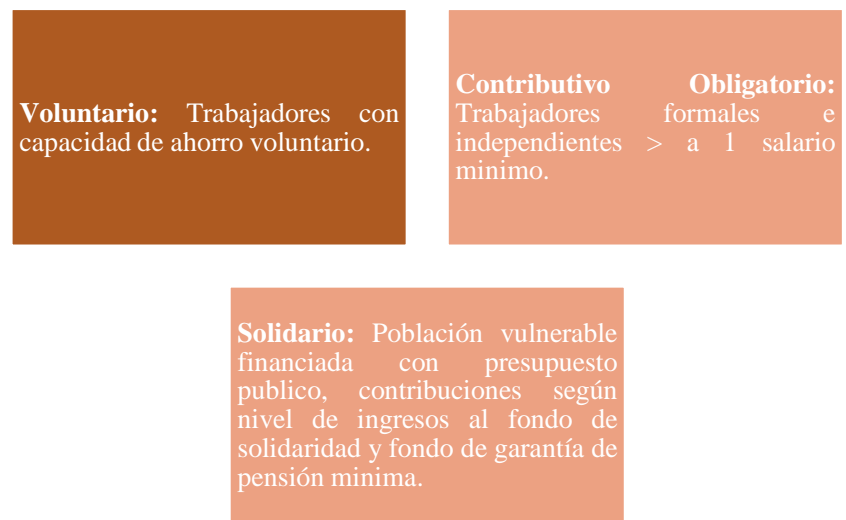

Figura 4. Pilares del Sistema Pensional en Colombia. Diagrama elaborado con base a la información de Asofondos. (Titze Menzel, 2012)

Estos pilares se administran de la siguiente forma:

- Ahorro voluntario

- Régimen de prima media

- Régimen de ahorro individual con solidaridad

- BEPS: Ahorro individual 


\section{- Colombia mayor}

Como ya se mencionó anteriormente, el sistema pensional colombiano permite que los afiliados decidan su permanencia en uno de los dos regímenes:

RPM: Régimen de Prima Media, el cual está a cargo del Instituto de Seguros Sociales y de las entidades previsionales que funcionaron antes de esta reforma y que hoy subsisten (CAJANAL, CAPRECON y algunos fondos). En este sistema se consagra lo que se denomina un "régimen de reparto", por medio del cual se constituye un fondo común público del que se hace responsable el Estado mismo, teniendo los aportantes al sistema garantizada una prestación definida luego de cumplidos los requisitos para acceder a la pensión (semanas de cotización, edad y en algunos casos fidelidad al sistema). (Garzón Trejos \& Varela Botero, 2017).

En este modelo los requisitos que se deben cumplir para la pensión de vejez se definen por semanas cotizadas, las cuales son: "mínimo 1300 a partir del 2015, mujeres 57 años y hombres 62 años, el monto de la pensión depende del ingreso base de cotización de los últimos 10 años cotizados. Para este régimen de reparto las cotizaciones de los trabajadores actuales pagan las mesadas de los pensionados" (Colpensiones, 2018).

RAIS: Régimen de ahorro individual con solidaridad, cen este sistema que se copió al modelo chileno, "se dejó en manos de los fondos privados de pensiones (AFP) la administración de los ahorros de los aportantes, de tal suerte que la pensión finalmente se "construye" con el ahorro en cuentas individuales que le pertenecen exclusivamente a él. Financieramente se trata de un régimen de capitalización plena con contribuciones no necesariamente definidas y prestaciones igualmente no definidas" (Garzón Trejos \& Varela Botero, 2017).

Bajo este régimen, el afiliado puede pensionarse antes de tiempo: solo debe acumular un volumen de capital que le permita financiar una pensión mensual equivalente al $110 \%$ del salario mensual mínimo legal vigente a 1993 actualizado en el IPC, sin que sea menor a un salario mínimo. De lo contrario, debe cumplir una edad determinada para acceder a la garantía estatal de pensión mínima (57 años para las mujeres, 62 para los hombres, 1.150 semanas cotizadas al cumplir dicha edad y no contar con otra fuente de ingresos). El afiliado, además, puede hacer aportes adicionales para aumentar la mesada pensional en el momento de la jubilación. En caso de fallecimiento, el capital ahorrado es heredable ( si no hay herederos de ley, puede entrar en sucesión hasta el quinto orden hereditario). La persona también puede tener mesada pensional vitalicia o escoger entre las modalidades de pensión en las AFP de Retiro Programado, Retiro Programado con Renta Vitalicia Diferida, Renta Temporal Variable con Renta Vitalicia Diferida, Renta Temporal Variable con Renta Vitalicia Inmediata, Retiro Programado sin negociación de Bono Pensional y Renta Vitalicia Inmediata. Si el afiliado tiene la capacidad de pensionarse con el máximo establecido por la ley, y 
además le sobra capital, la AFP le devolverá ese monto sobrante para que el trabajador disponga de él con total libertad. En caso de que no alcance a cumplir los requisitos para obtener su pensión, la AFP, que es vigilada por la Superintendencia Financiera de Colombia, le devolverá sus aportes junto con el valor del bono pensional, en caso de que haya. (Asobancaria, 2018).

Explicado lo anterior las principales diferencias entre el RPM y el RAIS son:

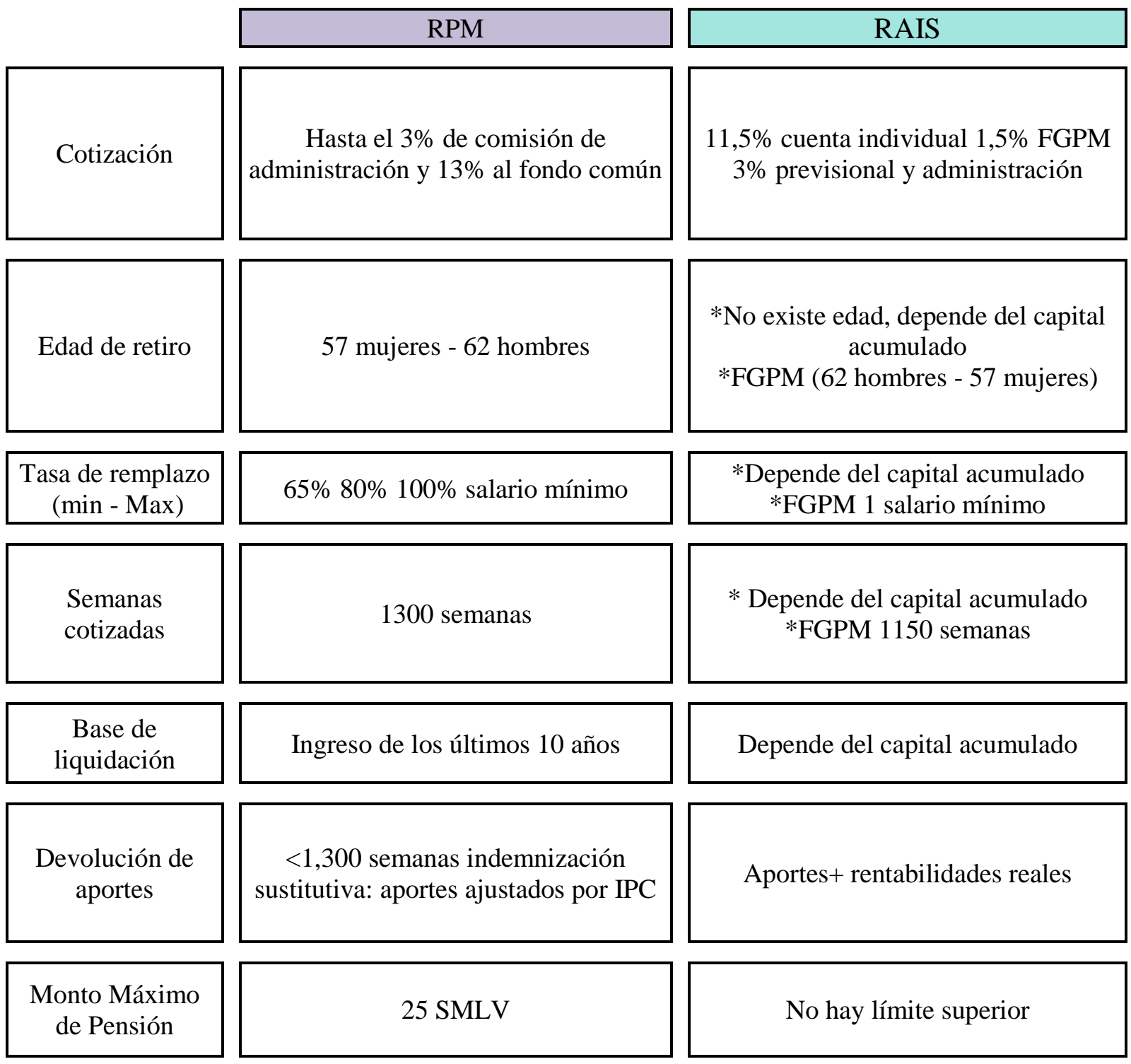

Nota: Elaboración propia (Acuña \& Suarez, 2018)

Tabla 4. Diferencias entre RPM y el RAIS 


\section{Tipos de pensión en Colombia por RAIS}

\begin{tabular}{|c|c|c|}
\hline $\begin{array}{l}\text { TIPO DE } \\
\text { PENSIÓN }\end{array}$ & DEFINICIÓN & REQUISITOS \\
\hline $\begin{array}{l}\text { PENSIÓN } \\
\text { POR } \\
\text { VEJEZ }\end{array}$ & $\begin{array}{l}\text { Corresponde al pago que recibe el afiliado al } \\
\text { concluir la vida laboral. Y es el resultado del ahorro } \\
\text { acumulado durante la etapa productiva (son los } \\
\text { aportes más los rendimientos generados), el cual es } \\
\text { depositado en una cuenta individual a nombre del } \\
\text { titular. }\end{array}$ & $\begin{array}{l}\text { Edad: } 57 \text { años las mujeres } \\
\text { y } 62 \text { los hombres } \\
\text { Capital: } 110 \% \text { del } \\
\text { SMLMV }\end{array}$ \\
\hline $\begin{array}{l}\text { PENSIÓN } \\
\text { POR } \\
\text { INVALIDEZ }\end{array}$ & $\begin{array}{c}\text { Es una prestación económica, en la que se paga una } \\
\text { renta mensual (pensión) y a aquella persona que ha } \\
\text { calificado como inválida y en la cual su enfermedad } \\
\text { o patología es de origen. }\end{array}$ & $\begin{array}{l}\text { Pérdida de capacidad } \\
\text { laboral > 50\% } \\
\text { Haber cotizado } 50 \\
\text { semanas durante los } \\
\text { últimos tres (3) años al } \\
\text { hecho que causó la } \\
\text { invalidez. } \\
\end{array}$ \\
\hline $\begin{array}{l}\text { PENSIÓN } \\
\text { POR } \\
\text { SOBREVIVENCIA }\end{array}$ & $\begin{array}{l}\text { Corresponde a una prestación económica de carácter } \\
\text { mensual que se encarga de cubrir a los beneficiarios } \\
\text { en caso de que el afiliado fallezca por razones } \\
\text { distintas a accidentes de trabajo o enfermedad } \\
\text { profesional, ya que para estos casos particulares son } \\
\text { las Administradoras de Riesgos Profesionales las } \\
\text { que se encargan de ofrecer la cobertura. }\end{array}$ & $\begin{array}{l}\text { El requisito que se debe } \\
\text { cumplir para que este tipo } \\
\text { de pensión se haga } \\
\text { efectiva, es que el afiliado } \\
\text { que fallece debe haber } \\
\text { cotizado por lo menos } 50 \\
\text { semanas durante los } \\
\text { últimos tres años a la } \\
\text { fecha de fallecimiento. }\end{array}$ \\
\hline
\end{tabular}

Nota: Elaboración propia (Acuña \& Suarez, 2018)

Tabla 5. Tipos de pensión en Colombia del RAIS

\section{Tipos de pensión en Colombia por RPM.}

Pensión por Vejez: Para tener derecho a la pensión por vejez el afiliado debe cumplir con los siguientes requisitos: mujeres 57 años y hombres 62 años y haber cotizado mínimo 1300 semanas.

Pensión de Vejez por Alto Riesgo: A esta pensión pueden acceder las personas que laboren en actividades de alto riesgo, por cada 60 semanas adicionales a las 1300 se le disminuye un año para pensionarse. Estas actividades son:

Actividades mineras en socavones o subterráneas.

* Los controladores aéreos.

* Personal de custodia de la guardia penitenciaria IMPEC.

* Personas que trabajan en altas temperaturas. 
Trabajadores en rayos ionizantes o rayos $\mathrm{x}$.

Trabajadores con gérmenes patógenos o sustancias altamente peligrosas.

Los bomberos y aquellos dedicados al control de incendios.

\section{Pensión De Vejez Anticipada Por Madre O Padre Cabeza De Familia Con Hijo} Discapacitado A Su Cargo: Si es madre o padre cabeza de familia y tiene un hijo en estado de invalidez, puede acceder a esta pensión. Así lo establece el parágrafo 4 del artículo 9 de la Ley 797 de 2003 (Congreso de la República de Colombia, 2003). Los requisitos que debe cumplir para el reconocimiento de esta pensión son:

* Ser madre o padre cabeza de familia y que los miembros de grupo familiar dependan del afiliado.

* Ser madre o padre de un hijo inválido que dependa de ti económica y socialmente.

* Haber cotizado el número mínimo de semanas exigidos para acceder a la pensión de vejez.

* El hijo menor o mayor de edad debe padecer una discapacidad superior al 50\% debidamente calificada.

* El hijo afectado por la invalidez física o mental debe permanecer en esa condición.

Pensión por Invalidez: El afiliado deberá ser declarado inválido por haber perdido el 50\% o más de su capacidad laboral, de acuerdo con las Juntas Regionales y/o Nacionales de Calificación de Invalidez, por consecuencia de una enfermedad de origen no profesional.

Pensión de Sobrevivientes: esta pensión es otorgada a quienes acrediten su calidad de beneficiarios como conyugue, compañero (a) permanente, hijos menores, hijos estudiantes entre 18 y 25 años, hijos inválidos o padres si dependían económicamente del afiliado o el pensionado.

Indemnización Sustitutiva: es el derecho al que tienen los afiliados cuando no cumplen con el requisito de semanas cotizadas para obtener una pensión ya sea de vejez, invalidez o sobrevivencia.

Debido a que en Colombia existen el sistema público y el sistema privado de pensión, para el RPM solo existe una modalidad de pensión que es Renta Vitalicia y para el Régimen de ahorro individual con solidaridad existen varias modalidades de pensión. Dentro de las -principales se encuentran: 


\section{Retiro Programado}

- La pensión será pagada por el Fondo de Pensiones, en este caso de acuerdo con los recursos acumulados en la cuenta de ahorro individual.

-El valor del incremento de la mesada pensional dependerá de los rendimientos generados en el Fondo de Pensiones.

-En caso de fallecimiento del pensionado, la pensión pasará a sus beneficiarios (Cónyuge, compañero (a) permanente, hijos con derecho y padres con derecho). Si no llegaran a existir beneficiarios de la pensión, el saldo de la cuenta de ahorro individual y el Bono Pensional harán parte de los bienes de la herencia.

\section{Renta Vitalicia Inmediata}

- La pensión será pagada por una Compañía de Seguros que previamente efectúa una cotización de la mesada, de acuerdo con las características del grupo familiar y del monto del capital que el afiliado tenga en su cuenta de ahorro individual

-El incremento anual de la mesada con el IPC

-En caso de fallecimiento del pensionado, la pensión pasará a sus beneficiarios (Cónyuge, compañero (a) permanente, hijos con derecho y padres con derecho).

\section{Retiro programado sin negociación de bono pensional}

- El afiliado se pensiona de manera anticipada a la fecha de redención del bono pensional emitido, bajo la modalidad de retiro programado descrita en el artículo 81 de la Ley 100 de 1993, sin necesidad de negociar el citado bono.

\section{Retiro Programado con Renta Vitalicia Diferida}

-Es una combinación entre las modalidades de pensión de Renta Vitalicia Inmediata y Retiro Programado.

-El valor de la mesada pensional debe ser uniforme en el tiempo de disfrute tanto en el retiro programado como en la renta vitalicia.

-El re cálculo de la pensión se hace cada año según la modalidad que se esté disfrutando: Retiro Programado de acuerdo con los rendimientos, Renta Vitalicia de acuerdo al IPC.

\section{Renta Temporal Cierta con Renta Vitalicia de Diferimiento Cierto}

-En este tipo de pensión se advierten dos etapas durante la vida pensional. La primera es por un período fijo denominado Renta temporal Cierta. La segunda fase es vitalicia, pues comprende el resto de la vida del pensionado o del último beneficiario de ley, y se llama Renta Vitalicia de Diferimiento Cierto.

Figura 5. Modalidades de pensión (Colpensiones, 2018).

\section{Momento Actual en Colombia}

Actualmente los Fondos de Pensiones en Colombia se han convertido en una fuente de ahorro interno para el país como se ve en la figura 6 (Superintedencia Financiera de Colombia, 2018) 
generando rendimientos para sus afiliados estimados en un $62 \%$ del ahorro pensional correspondientes a un 25\% del PIB (Producto interno bruto)., (Asofondos, 2018)

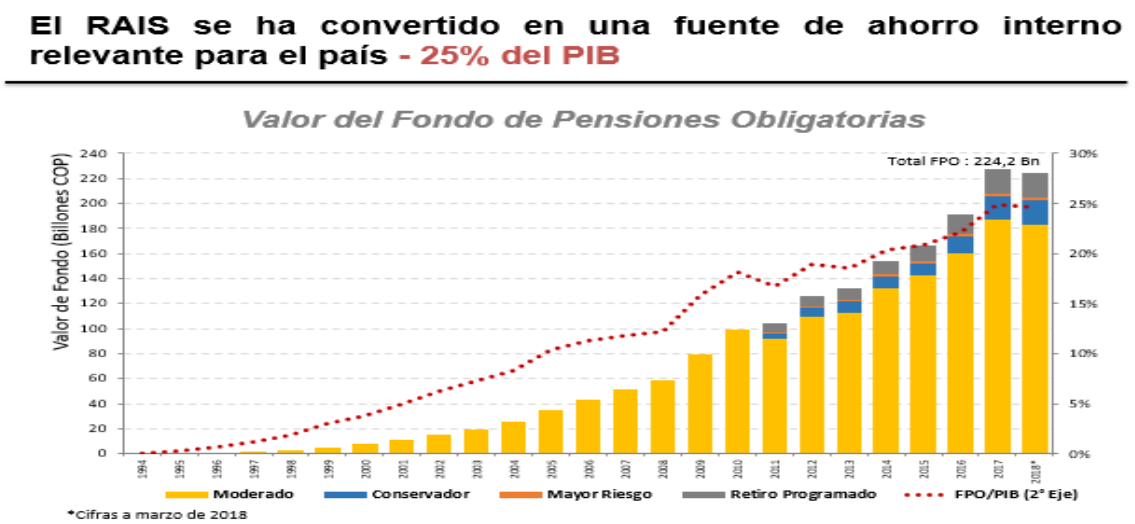

Figura 6. Valor de los fondos de pensiones en Colombia. Datos proporcionados por Superintendencia Financiera de Colombia (Superintedencia Financiera de Colombia, 2018).

Es por esto que para que el sistema pensional en Colombia sea viable, el gobierno propone generar una reforma pensional donde se puedan llenar los espacios vacíos que amenazan las finanzas del estado. (Asofondos, 2018)

Dentro de las acciones que demandan la urgencia de una reforma se encuentran:

Falta de Cobertura: frente a la región y realizando una comparación a nivel mundial, Colombia presenta uno de los niveles más bajos de cobertura pensional llegando solo al 30\% de su población cuando el promedio en América Latina es del 64\%.

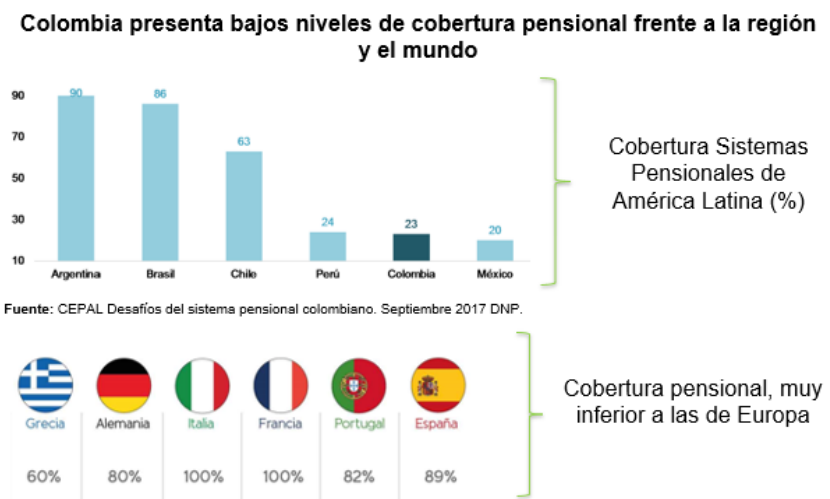

Figura 7. Cobertura pensional colombiana frente a la región. Fuente: (OECD., 2016) 
Insostenibilidad fiscal e inequidad: de acuerdo con las cifras presentadas por la Superintendencia financiera cada año se destinan cerca de 34 billones de pesos para el pago de las pensiones del sistema público (RPM) donde la cifra de pensionados es de 1.3 millones de personas, las cuales se llevan aproximadamente el $85 \%$ de los recursos anteriormente mencionados.

\section{De hecho, el sistema de reparto no es compatible con un aumento de formalidad, entre mayor formalidad mayor déficit pensional.}

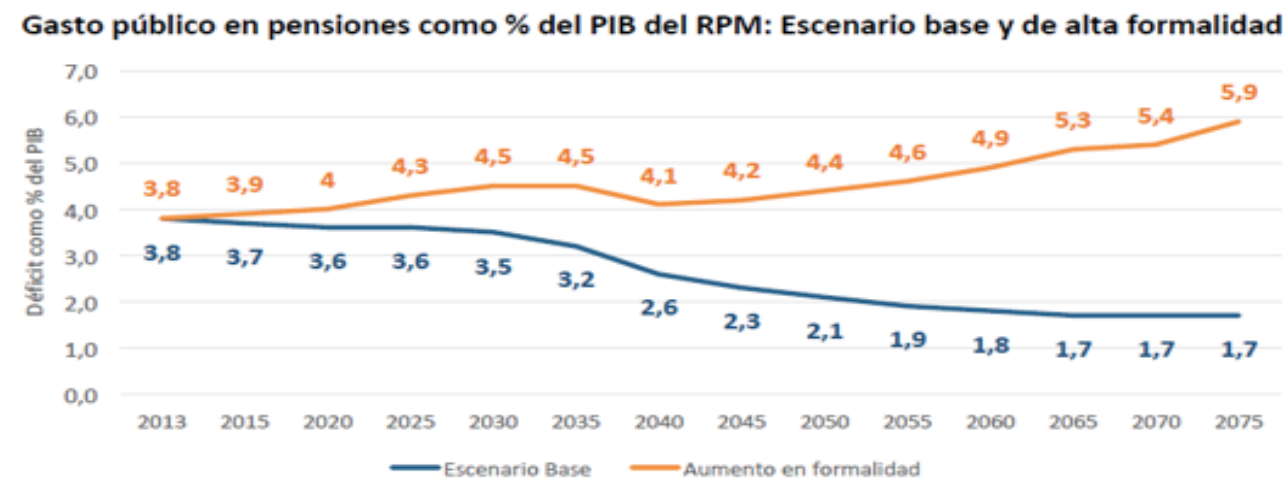

Figura 8. Número de pensionados por RPM. Fuente: Superintendencia financiera de Colombia (Financiera, 2018)

\section{PENSIONAOOSENCOLOMBIA}

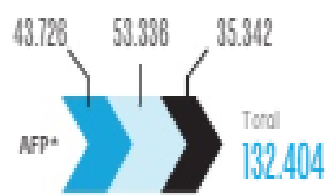

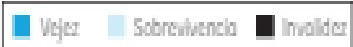

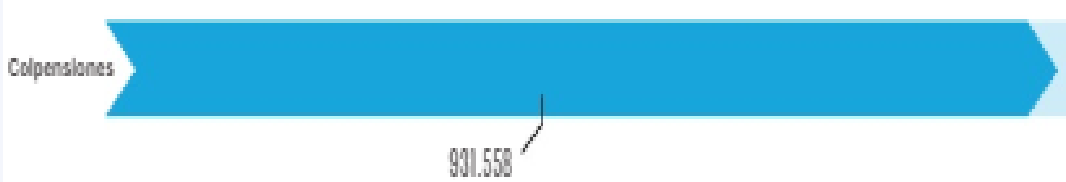

Figura 9. Porcentaje de gasto pensional RPM. Fuente: (Desarrollo \& Financiera, 2018)

De continuar con este esquema, las consecuencias serían una desistimulación del ahorro y un agrandamiento del déficit para cubrir el pago de las mesadas pensionales, debido a que muchas de estas mesadas están cubiertas por subsidios desmedidos e injustificados ofrecidos por el estado como se puede evidenciar en la 
Los subsidios pensionales están dirigidos a las personas con ingresos más altos SUBSIDIOS A PENSIONADOS DE COLPENSIONES POR RANGOS DE INGRESOS

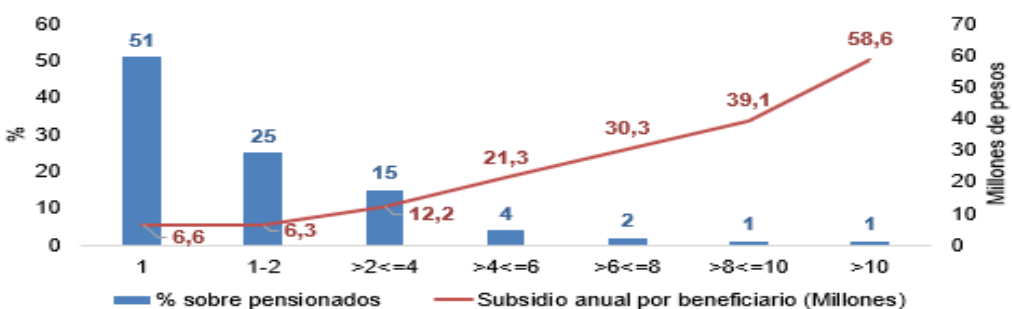

$\mathbf{8 6} \%$ de los subsidios del régimen público se dirigen al $\mathbf{2 0} \%$ de la población con ingresos más altos.

El quintil de población con ingresos más bajos sólo recibe el 0,1\% de los subsidios pensionales.

Figura 10. Presupuesto pensional. Fuente: Superintendencia Financiera (Financiera, 2018)

Pensiones del sistema público absorben muchos recursos al ser subsidiados y tienen una baja cobertura (gasto altamente regresivo)

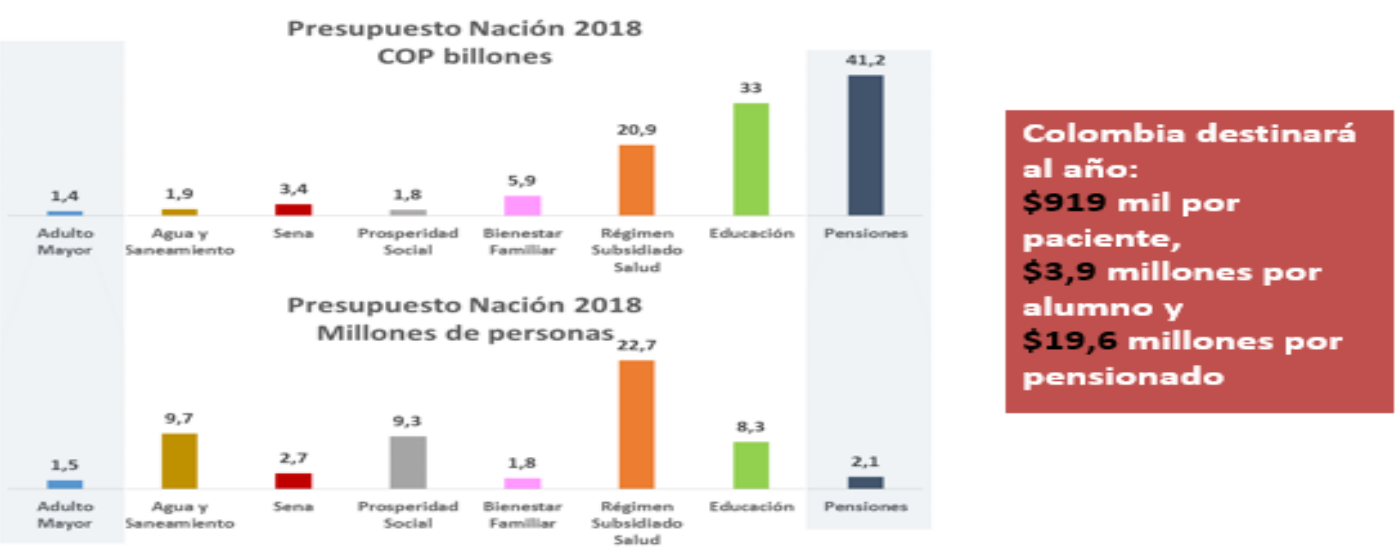

Figura 11. Subsidios pensionales. Fuente: Ministerio de Hacienda y Crédito Público.

El régimen de ahorro individual ha contribuido fundamentalmente a la sostenibilidad fiscal

VPN de Deuda Pensional implícita $\% P I B$

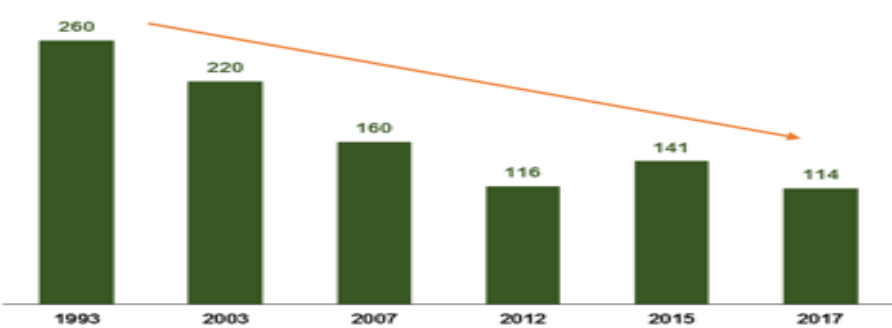

Figura 12. Sostenibilidad Fiscal. Fuente: Ministerio de Hacienda y Crédito Público. 
Por otro lado, después de realizar el estudio se evidencia que el régimen de ahorro individual ha contribuido a la sostenibilidad fiscal desde el momento del ingreso de las AFP al sistema pensional.

Por lo anterior las propuestas realizadas por Asofondos y Fedesarrollo y el Gobierno Nacional, van desde no tocar las edades de pensión actual (57 años Mujeres y 62 Hombres) o que estas edades se igualen para que hombres y mujeres se jubilen al tiempo, hasta que Colpensiones (Entidad que maneja el RPM en Colombia) opere dentro de los parámetros y el esquema de las AFP (Fondos privados).

\section{Arquitectura de un sistema Pensional Sostenible}

\section{COMPARATIVO ENTRE PROPUESTAS DE REFORMA PENSIONAL}

\begin{tabular}{|c|c|c|c|}
\hline Propuesta & Asofondos & Comisión del Gasto & Fedesarrollo \\
\hline $\begin{array}{c}\text { Prioridad de una Reforma } \\
\text { Pensional }\end{array}$ & Alta & Alta & Alta \\
\hline Tipo de Sistema & $\begin{array}{l}\text { Fondo de Reparto + } \\
\text { Capitalización Individual }\end{array}$ & $\begin{array}{c}\text { Sistema multipilar } \\
\text { (Fedesarrollo) / } \\
\text { Capitalización Individual }\end{array}$ & $\begin{array}{c}\text { Reparto por } 1 \text { SM L para todo } \\
\text { trabajador, Capitalización } \\
\text { por el excedente del ingreso } \\
\text { (multipilar) }\end{array}$ \\
\hline Ingreso Base Liquidación & $\begin{array}{l}\text { Prom edio del salario durante } \\
\text { toda la vida laboral }\end{array}$ & $\begin{array}{l}\text { Promedio del salario durante } \\
\text { toda la vida laboral }\end{array}$ & \begin{tabular}{|c|} 
Promedio del salario durante \\
toda la vida laboral. Dis minuir a \\
1.150 s emanas para pens ión.
\end{tabular} \\
\hline $\begin{array}{l}\text { Aumentar la edad de } \\
\text { Pensión }\end{array}$ & No se requiere & $\begin{array}{l}\text { Si. Igualar entre hombres y } \\
\text { mujeres, y realizar ajustes } \\
\text { periódicos con parámetros } \\
\text { demográficos }\end{array}$ & $\begin{array}{c}\text { Si. Aumentar a } 65 \text { años e } \\
\text { igualar entre hombres y } \\
\text { mujeres }\end{array}$ \\
\hline Porcentaje de Cotización & Aumento gradual progresivo & Aumentar cotización al $18 \%$ & Aumentar cotización al $18 \%$ \\
\hline $\begin{array}{l}\text { Fondo Garantia Pensión } \\
\text { Minima }\end{array}$ & $\begin{array}{c}\text { Complemento para otorgar las } \\
\text { pensiones minimas (Fondo de } \\
\text { Reparto) }\end{array}$ & $\begin{array}{l}\text { Aumentar proporcionalmente } \\
\text { los aportes a FGPM con } \\
\text { aumento en cotización / } \\
\text { Eliminar los aportes al FGPM }\end{array}$ & $\begin{array}{c}\text { Eliminar los aportes al FGPM y } \\
\text { al FSP }\end{array}$ \\
\hline
\end{tabular}




\section{COMPARATIVO ENTRE PROPUESTAS DE REFORMA PENSIONAL}

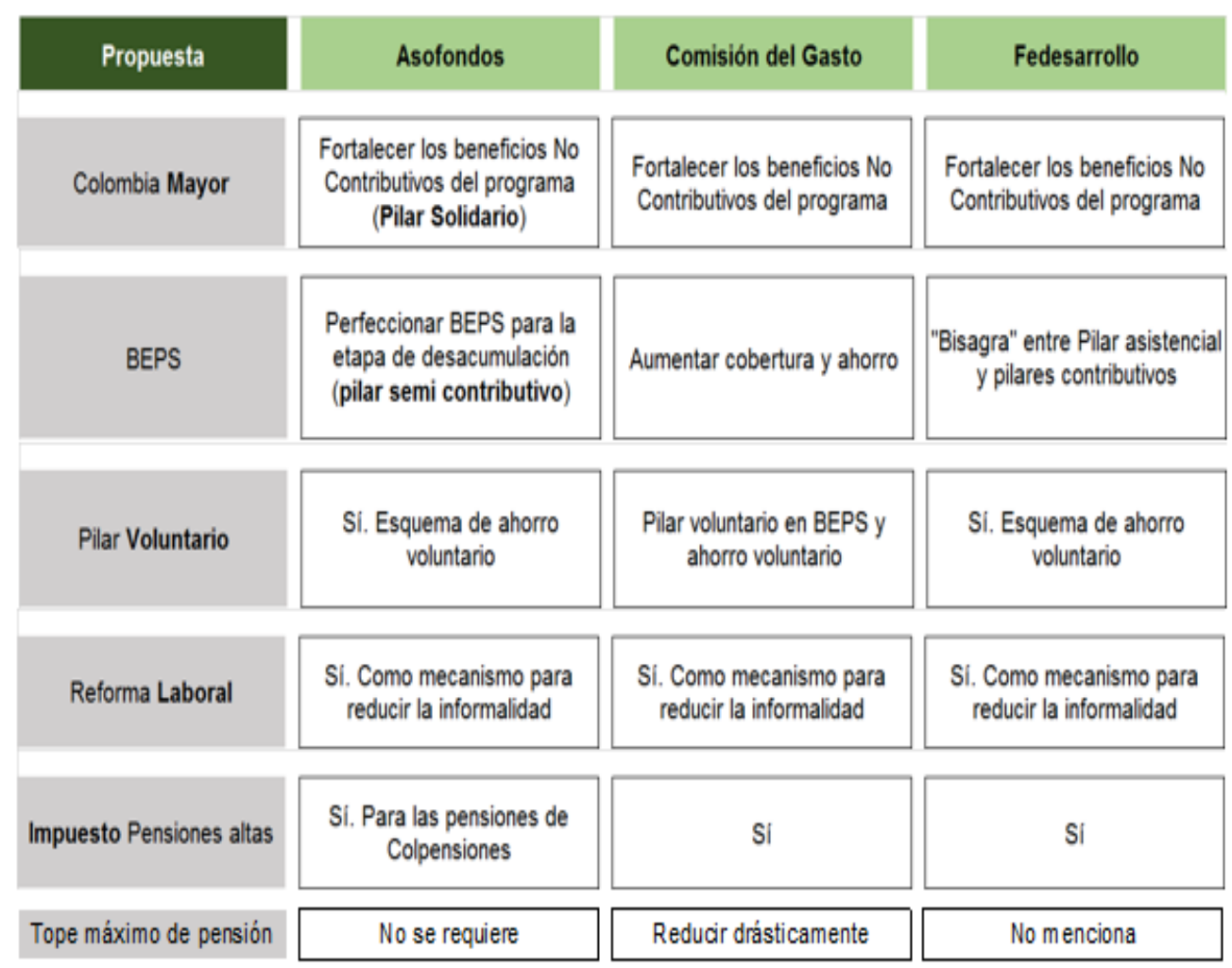

Figura 13. Estructura pensional propuesta. Fuente: Asofondos (2018)

\section{Arquitectura para un Sistema Pensional sostenible}

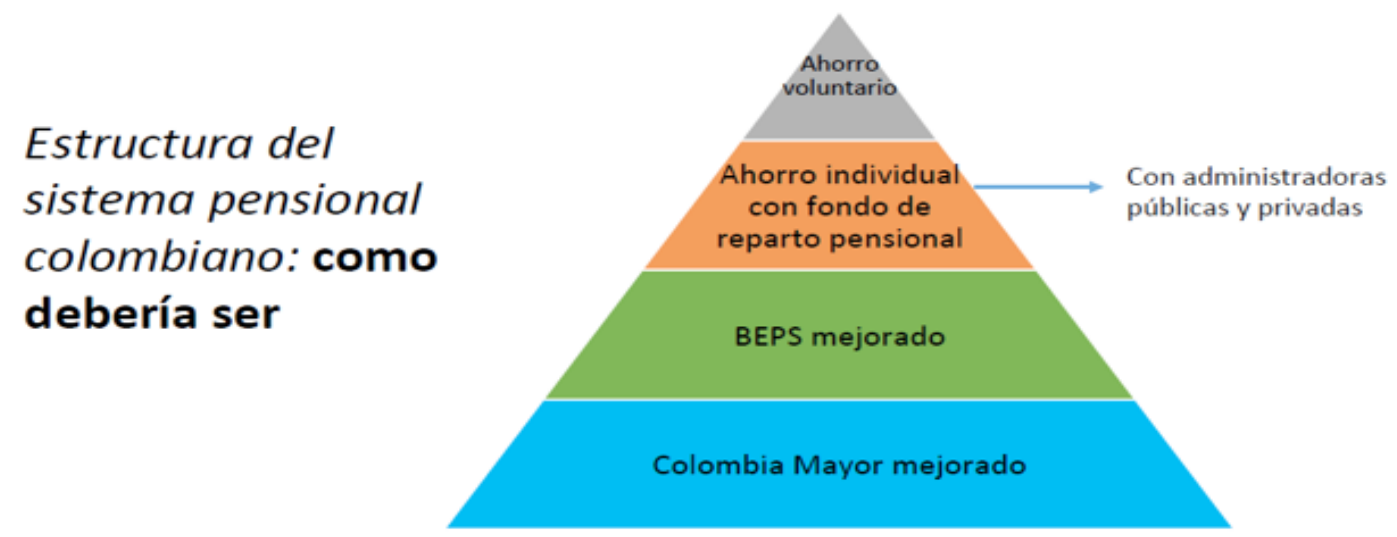

Figura 14. Comparativo de la reforma pensional propuesta. Fuente: Asofondos. 
La obligación de la reforma pensional es mejorar el ahorro y no cargar al trabajador formal la responsabilidad de subsidiar a aquellas personas que se encuentran en la informalidad.

\section{Momento Actual en Chile}

Como en todos los países de la región y a pesar de que Chile es pionero en seguridad social es necesario la evaluación de una reforma pensional debido al hueco fiscal que se está generando.

El gobierno del presidente Sebastián Piñera está evaluando como realizar una reforma que genere cobertura, sostenibilidad y equidad. De acuerdo con el experto pensional Augusto iglesias consultor y experto en seguridad social, aconseja que el sistema pensional chileno le urge un ajuste ya que es un país que no puede confiar en el componente de prima media, debido a que la estabilidad financiera se deteriora con el envejecimiento de la población por lo tanto el sistema pensional debe moverse hacia los sistemas de ahorro (Bolaños, 2018).

Con las reformas pensionales, Chile busca sustituir su sistema de reparto por uno de capitalización individual, sin embargo, esto representa un desafío para el sistema fiscal.

La lógica del régimen de reparto, es considerar a la generación pensionada como acreedora de una deuda del Estado, por sus contribuciones al sistema cuando eran activos. El Estado financia el pago de esta deuda mediante una nueva deuda que proviene de las cotizaciones de la generación activa actual. Al traspasar ahora las cotizaciones de los trabajadores a las administradoras privadas de fondos de pensiones, la reforma viene a limitar la capacidad del Estado para financiar una deuda previsional con otra. Lo obliga en cambio a hacer explícita una deuda que debe amortizar mediante otras fuentes del presupuesto fiscal. (CEPAL. Sección de Estudios del Desarrollo, 2014)

De acuerdo con lo investigado las decisiones más relevantes para llevar a cabo la reforma chilena son: la consolidación de su cobertura poblacional, el importante esfuerzo fiscal por financiar la transición, operación de un mercado imperfecto donde se manifiestan importantes costos de operación, y si bien ha contribuido a impulsar el desarrollo del mercado de capitales, su efecto directo sobre la inversión productiva es limitado. 


\section{Conclusiones Colombia}

La pregunta más común en la actualidad pensional para el ciclo más joven del mercado laboral colombiano es ¿Podre alcanzar mi pensión? Esta es una duda que se genera a largo y mediano plazo y que tiene como factor principal los problemas provocados por las fallas en la reglamentación que no permiten la fidelidad del sistema y que ponen en evidencia los problemas fiscales del sector.

Como opinión de los autores, el régimen pensional de Ahorro individual con solidaridad (RAIS) es un modelo que cuenta con los recursos propios y suficientes para el pago de sus obligaciones pensionales actuales y a futuro.

Mientras que por otro lado el régimen de prima media (RPM) trae consigo una responsabilidad fiscal mayor debido a que la deuda genera un hueco en las finanzas del estado al ser una bolsa común que trae una deuda heredada de cada ciclo laboral anterior. Cada nuevo pensionado en este régimen va minimizando las reservas para el pago de las obligaciones futuras volviéndose de alguna manera inviable para el país.

Actualmente el gobierno debe tomar medidas en caso de generar y aprobar nuevas reformas donde se debe tener como eje principal la disminución del desempleo, adicionalmente se debe trabajar en una cultura de ahorro donde todos los colombianos que inician su vida laboral entiendan la importancia de este ejercicio.

\section{Conclusiones Chile:}

Como consecuencia de que el sistema pensional del Chile presentaba varias falencias y se comenzaba a evidenciar el aumento de esperanza de vida de las personas mayores, consistencia de cotizaciones, entre otros se vio la necesidad de crear las AFP.

Es importante resaltar que el gobierno tiene que conseguir y mantener la secuencia laboral, la formalización para el empleo, mejorar el capital humano e incrementar la productividad.

Si se deseara mejorar las pensiones en un largo plazo se podría realizar cambios relevantes en los requisitos para pensionarse como: aumentar la edad de pensión, realizar un seguimiento de los afiliados que cotizan como independientes, generar una cultura de ahorro voluntario y un aumento en el aporte que genera el empleador. 
Luego de toda la información que se pudo observar, se concluye que el Sistema pensional chileno no es mejor ni peor que el Sistema pensional colombiano. Cada uno tiene sus fortalezas y debilidades muy propias de sus distintas condiciones sociales, económicas y políticas. 


\section{Referencias}

Banco Interamericano de Desarrollo. (s.f.).

2018, A. (s.f.).

Asamblea General de la ONU. (10 de 12 de 1948). Declaración Universal de Derechos Humanos. articulo 22. Paris, Francia: Naciones Unidas.

Asofondos. (s.f.). Obtenido de www.asofondos.org.co

Biblioteca del Congreso Nacional de Chile. (1 de Abril de 2013). BCN. Obtenido de Guía legal sobre fondos de pensiones: https://www.ben.cl/leyfacil/recurso/fondos-de-pensiones

Bolaños, L. F. (2018 de julio de 2018). LA REPÚBLICA. Obtenido de https://www.larepublica.co/finanzas/todas-las-reformas-que-se-han-hecho-buscanalejarse-de-la-prima-media-afirma-augusto-iglesias-2746039

CENDA. (s.f.). Análisis comparativo de los sistemas de seguridad social en Chile, Colombia.

CEPAL. Sección de Estudios del Desarrollo. (2014). www.cepal.org. Obtenido de Reforma al sistema de pensiones chileno: https://www.cepal.org/es/publicaciones/5221-reforma-alsistema-pensiones-chileno

Chile, A. A. (s.f.). Obtenido de https://www.aafp.cl/el-sistema/el-sistema-afp/antiguo-sistema/

Colpensiones. (s.f.). Obtenido de www.colpensiones.gov.co

Colpensiones. (5 de abril de 2018). Pensiones y afiliaciones. Obtenido de Tipos de pensión y afiliación:

https://www.colpensiones.gov.co/pensiones/Publicaciones/proximos_a_la_pension/Pensi on/tipos_de_pension_y_otras_prestaciones

Comisión Económica para América Latina y el Caribe- CEPAL. (s.f.). Obtenido de www.cepal.org/es

Congreso de la República de Colombia. (2003). Ley 797 de 2003. Recuperado el 4 de 9 de 2018, de http://bibliotecadigital.ccb.org.co/handle/11520/13860

Croquevielle, T. (02 de 08 de 2016). Pensiones en el mundo, sus orígenes y diferentes modelos. El definido.

Davivienda. (17 de abril de 2018). Mis finanzas para invertir. Obtenido de https://www.misfinanzasparainvertir.com/noticias/los-distintos-modelos-pensionales-enel-mundo/

Desarrollo, B. I., \& Financiera. (2018). 
Fedesarrollo. (12 de abril de 2010). www.repository.fedesarrollo.org.co. Obtenido de www.fedesarrollo.org.co:

https://www.repository.fedesarrollo.org.co/bitstream/handle/11445/351/El-sistemapensional-en-Colombia_Retos-y-alternativas-para-aumentar-la-cobertura-12-de-abril2011.pdf?sequence=1

Financiera. (2018).

Franco, P., Oquendo, M., \& Berrio, C. (2015). Posibles perspectivas del sistema de pensiones en Colombia. Revista CES, 79-93.

Garzón Trejos, D., \& Varela Botero, J. (2017). Garantias Constitucionales de los Regimenes pensionales en Colombia. Bogotá: Universidad Libre seccional Cali .

Ley No 20255 (Diario Oficial de la República de Chile, Santiago, Chile (Ministerio del Trabajo y Previsión Social de Chile 11 de Marzo de 2008).

Mesa-Lago, C. (1991). "Social Security in Latin America". Informe elaborado para IADB, Economic and Social Progress in Latin America, Report.

Ministerio de Hacienda y Crédito Público. (s.f.). Obtenido de www.minhacienda.gov.co

Navarro Wolf, A. (s.f). Asofondos. Obtenido de ¿quien se pensiona en Colombia?: http://www.asofondos.org.co/sites/default/files/xmemorias/Antonio_Navarro.pdf

OECD. . (Octubre de 2016). Comisión Europea y Administración de la Seguridad Social en Estados Unidos. Recuperado el Agosto de 2018, de Perspectivas de la OCDE: http://www.oecd.org/sti/ieconomy/DigitalEconomyOutlook2015_SP_WEB.pdf

OIT. (1996-2018). www.ilo.org/global/lang--es/index.htm.

Porvenir. (s.f.). Obtenido de www.porvenir.com.co

RANGEL, K. T. (2017). EVOLUCIÓN DEL MODELO PENSIONAL EN COLOMBIA. Obtenido de Facultad de Derecho, Universidad Libre, 159.

Rankia. (s.f). Fondos de pensiones AFP. Recuperado el septiembre de 2018, de https://www.rankia.cl/blog/fondos-pensiones-afp/3236972-cuales-son-tipos-modalidadespension

Suarez, A. \&. (2018).

Superintedencia Financiera de Colombia. (21 de septiembre de 2018). Pensiones y Cesantias. Obtenido de https://www.superfinanciera.gov.co/jsp/loader.jsf?1Servicio=Publicaciones\&lTipo=public aciones\&lFuncion=loadContenidoPublicacion\&id=61153

Superintendecia Financiera de Colombia. (s.f.). Obtenido de www.superfinanciera.gov.co 
Superintendencia de Pensiones . (s.f.). Obtenido de www.spensiones.cl

Titze Menzel, M. (15 de marzo de 2012). Asofondos. Obtenido de Pensiones solidarias en ChileLecciones para Colombia: http://www.asofondos.org.co/sites/default/files/Pensiones\%20Solidarias\%20en\%20Chile. pdf

Universidad del Valle, L. C. (12 de 2012). bibliotecadigital.univalle.edu.co. Obtenido de EL SISTEMA PENSIONAL COLOMBIANO: UN ANALISIS A LOS DETERMINANTES DE LA AFILIACION Y SU PAPEL EN EL IMPACTO DE LAS REFORMAS PENSIONALES:

http://bibliotecadigital.univalle.edu.co/bitstream/10893/5957/1/0461683-p.pdf

Universidad, Libre. (2017). Facultad de Derecho. 